\title{
Atuação do Profissional de Educação Física em uma Instituição de Longa Permanência para Idosos: um relato de experiência
}

\author{
Performance of the physical education professional in a Long \\ Term Care Institution for the Elderly: an experience report
}

MARTINEZ, V. M. L.

Especialização em Neurociências e Comportamento e mestrando do Programa de Pós-Graduação em Psicologia na Pontifícia Universidade Católica do Rio Grande do Sul (PUCRS). E-mail: victormatheusIm@hotmail.com

DOI: https://doi.org/10.53817/1983-6929-ragg-v12n1-4

Resumo: Introdução: A atuação de uma equipe multidisciplinar em saúde, com a inclusão dos serviços de Educação Física, é essencial no cuidado ao longo da vida e, sobretudo na senilidade. Objetivos: Relatar a experiência de atuação do Profissional de Educação Física em uma Instituição de Longa Permanência para Idosos (ILPI) na cidade de Porto Alegre/RS. Metodologia: Para tanto, foi realizado um estágio observacional junto ao profissional de Educação Física da instituição durante um (1) mês em horário matutino, entre os dias 4 a 29 de novembro de 2019, por um (1) ou dois (2) turnos semanais (segunda e/ou quarta-feira). Além da observação, utilizou-se buscas de dados na literatura cientifica, a partir de uma revisão narrativa bibliográfica. Resultados: A observação da realidade, contraposta pela literatura, revelou que os idosos necessitam de um estímulo constante quanto às atividades físicas de modo a manterem sua funcionalidade, mobilidade e saúde cognitiva. Conclusão: A atuação do Profissional de Educação Física junto a equipes multiprofissionais em saúde, neste contexto, é extremamente necessária e, portanto, esses profissionais devem ser incluídos cada vez mais a atuar nos serviços da área de forma multiprofissional dentro das ILPIS.

Palavras-chave: Educação Física; ILPIS; Idoso.

\section{Abstract:}

Introduction: The performance of a multidisciplinary team in health, with the inclusion of Physical Education services, is essential in lifelong care and especially in old age. Objectives: To report the experience of the performance of the Physical Education Professional in a Long-Term Care Institution for the Elderly (LCFE) in the city of Porto Alegre/RS. Methodology: For this purpose, an observational internship was carried out with the institution's Physical Education professional for one (1) month in the morning, between November 4th and 29th, 2019, for one (1) or two (2) shifts weekly (Monday and/or Wednesday). In addition to observation, data searches in the scientific literature were used, based on a narrative bibliographic review. Results: The observation of reality, contrasted by the literature, revealed that the elderly need a constant stimulus regarding physical activities in order to maintain their functionality, mobility and cognitive health. Conclusion: The performance of the 
Physical Education Professional with multidisciplinary teams in health, in this context, is extremely necessary and, therefore, these professionals must be increasingly included to work in the area in a multidisciplinary manner within the LCFE.

Key words: Physical Education; LCFE; Elder.

\section{INTRODUÇÃO}

O envelhecimento humano é algo inevitável, e com o envelhecimento cronológico do passar dos anos se envelhece o sistema nervoso como um todo, ou seja, a idade biológica aparecerá inevitavelmente, e seus efeitos podem em muitos casos não serem atrativos para a população senil (WOLF, 2009; DZIECHCIAŻ; FILIP, 2014; ANDREASSEN et al., 2019).

A população senil cresce de modo exponencial em nosso país, e segundo o Instituto Brasileiro de Geografia e Estatística (IBGE) (2018), por meio da Pesquisa Nacional por Amostra de Domicílios Contínua (PNAD), o Brasil apresentou um aumento cerca de 4,8 milhões de idosos desde 2012, tendo assim um crescimento de forma linear em todos os estados da nação, o que representa um aumento de $18 \%$ na totalidade dessa faixa etária, totalizando $14,6 \%$ da população brasileira. Cabe ainda ressaltar que o estado do Rio Grande do Sul (RS), juntamente com o estado do Rio de Janeiro (RJ), apresenta os maiores índices percentuais de pessoas com idade igual ou superior a 60 anos (18,6\%).

Consequentemente, com o avanço da população idosa no Brasil, surgem as preocupações com os cuidados, no que diz respeito ao bem-estar físico, mental e social, tendem a aumentar na mesma proporção, de modo que esse envelhecer populacional seja da forma mais saudável possível (BRASIL, 2020).

O processo do envelhecimento indubitavelmente a maioria das pessoas que hoje são jovens passarão, a "terceira idade", a qual muitas vezes é algo temido, principalmente pelas associações com as doenças que porventura possam ser desencadeadas nesta etapa da vida, em especial as doenças crônicas não transmissíveis (DCNTs), como doenças neurodegenerativas, cardiopulmonares, etc. Durante o processo de mudanças ocasionados pelas DCNTs há um maior comprometimento das funções mentais e físicas, e essas mudanças podem ser minimizadas com a inclusão da prática das Atividades Físicas (AF) de forma ativa (DI LIEGRO et al., 2019). 
Porém, mesmo que se saiba dos benefícios da AF regular, o nível de AF está diminuindo na população brasileira. Algo que é atestado por Guthold et al. (2018), os quais fizeram um estudo global entre 2001 e 2016 sobre os níveis atividade física, e identificaram que Brasil é o país da américa latina com maior nível de sedentarismo, 47\% da população não pratica atividade física nos níveis mínimos recomendados. Dados esses que em idosos significa uma séria ameaça para o organismo destes indivíduos, estimulando assim o surgimento de patologias crônico-degenerativas, transtorno de humor, diminuindo as funções fisiológicas e cognitivas. Esses dados se sustentam, pois, em estudo recente do IBGE, mais de $40 \%$ dos adultos brasileiros apresentam-se inativos fisicamente (IBGE, 2020).

A importância das práticas corporais é tanta, que o Ministério da Saúde (MS), com base na Política Nacional de Promoção da Saúde (2006), tem entre seus macros objetivos a promoção da qualidade de vida por intermédio da atividade física, gerando hábitos saudáveis na população, de modo a reduzir os riscos à saúde (BRASIL, 2006).

De acordo com Chan (2015), Diretora da Organização Mundial da Saúde (OMS), a perda das habilidades comumente associada ao envelhecimento na verdade está apenas vagamente relacionada com a idade cronológica das pessoas, não existe um idoso típico, o envelhecimento saudável é mais que apenas a ausência de doença.

Nesse sentido, a forma como o indivíduo gerencia sua vida e suas vivências influenciarão diretamente no idoso que se tornará, ou seja, as atividades desempenhadas ao decorrer de sua vida resultarão em um envelhecer bemsucedido ou não. Nesse ponto a AF na terceira idade torna-se primordial, e algo que deve ser estimulado ao longo da vida, pois além de melhorar os parâmetros físicos ao envelhecer, auxilia na manutenção da atividade cognitiva, metabólica, psicossocial, e colabora para a redução dos riscos de queda, problema eminentes nesta etapa da vida (OKUMA, 1997; NELSON et al., 2007; LOURENÇO et al., 2019; OMS, 2019).

Quanto à atividade física executada em instituições de longa permanência para idosos, os ILPIS, as evidências científicas nos apresentam que tais intervenções devem ser enfatizadas com veemência, pois além de melhor o condicionamento mental e principalmente físico dos idosos, impedindo regressões no que tange a mobilidade articular e até promovendo ganhos consideráveis nesse 
âmbito, a prática regular de exercícios físicos ajuda a evitar quedas, o que na senescência é algo extremamente perigoso tendo em vista as comorbidades associadas à idade, como a osteoporose (diminuição progressiva da densidade óssea) (COUGHLAN; DOCKERY, 2014; HEWITT et al., 2018).

Considerando as progressões dos dados sobre 0 envelhecimento populacional e a importância da prática de atividade física ao longo da vida de forma ativa, em especial no período senil, encontra-se a importância do seguinte relato de experiência. Portanto, o objetivo desta observação foi identificar a práxis diária do profissional de Educação Física no contexto geriátrico em uma ILPI.

\section{METODOLOGIA}

O presente estudo é de característica descritiva, com base no relato de experiência de forma observacional. Portanto, foi realizado um estágio de observação pelo período de um (1) mês no local, sempre no período da manhã (1 a 2 turnos semanais) de modo a compreender a realidade da clínica geriátrica. Além disso, para compor os dados obtidos em campo, bem como melhor compreendê-los, se realizou uma pesquisa na literatura de cunho narrativo.

O estudo foi realizado durante o estágio em uma Instituição de Longa Permanência para Idosos (ILPI) situada na cidade de Porto Alegre/RS, no período de 4 a 29 de novembro de 2019, onde acompanhei a rotina do Profissional de Educação Física o qual atua no "Residencial Geriátrico Com Vivência". O ILPI é dirigido por 3 profissionais da área da saúde, sendo 1 médico e 2 psicólogas. As informações sobre a clínica e o contexto histórico foram de difícil acesso, sendo o breve resumo relatado principalmente pelo profissional de Educação Física e pela profissional competente pela administração.

O Residencial Geriátrico Com Vivência tem uma gama de aparatos médicos e profissionais. As instalações são distribuídas em 4 andares, nos quais residem 52 idosos (sendo 22 mulheres e 30 homens) com faixa etária entre 60 e 90 anos. A ILPI, conta com andares de sala de ginástica, espaço de lazer, espaço para recepção/reunião de familiares, e um posto de enfermagem no $3^{\circ}$ andar da casa.

A infraestrutura do local é adequada, além de localizar-se em um bairro calmo de uma realidade socioeconômica alta, sendo, portanto, a maioria seus moradores pertencentes a esta realidade. $O$ valor da mensalidade fica entre 7 e 10 salários 
mínimos do Brasil a depender do estado de saúde do residente e dos cuidados que cada idoso requer.

Quanto aos recursos humanos da clínica são multiprofissionais distribuídos em aproximadamente 40 técnicos de enfermagem (que se dividem nos três turnos), além de contar com diversos graduados na área da saúde, como: Fonoaudiologia, Farmácia, Terapia Ocupacional, Fisioterapia (3 profissionais), Medicina (diversas áreas), Nutrição, Psicologia e Educação Física.

Os profissionais do local relataram que a maioria dos moradores que procuram atendimento na clínica apresentam Doenças como de Acidente Vascular Cerebral (AVC) e Doença de Alzheimer, dentre outros problemas neurais e motores.

\section{RESULTADOS E DISCUSSÃO}

Os profissionais que atuam na clínica dividem-se nos 3 turnos, normalmente trabalhando em média 6 horas, logo como o estágio observacional se deu unicamente no período da manhã. Normalmente no período da manhã existem 1 fisioterapeuta, 1 fonoaudiólogo, técnicos de enfermagem espalhados pelos andares (aproximadamente 10) e claro, 1 profissional de Educação Física.

Conforme relatado pelo Profissional de Educação Física, a rotina dos idosos são diversas: acordam em média entre 7 e 8 horas da manhã, tomam banho e logo em seguida dirigem-se para a mesa do café, alimentam-se, e após isso eles sentam na sala de estar presente em cada andar. No entanto, mesmo com esse período pós café onde sentam-se nas salas de estar de cada andar (entre 9 e 10 horas da manhã), pude perceber que não há interação entre eles na maioria dos casos, exceto quando estimulados por algum profissional (no caso dos residentes da Unidade de Cuidados Especiais (UCE), eles pouco saem de suas respectivas camas).

Muitos dos idosos que ali residem encontram-se debilitados cognitivamente e por isso não desenvolvem diálogos entre si, também apresentando dificuldade de interação com os profissionais da clínica, ou se desenvolvem alguma conversa, é com relatos incoerentes ao assunto previamente tratado (sintomas da Doença de Alzheimer e outras doenças neurodegenerativas). Fatos empíricos estes que colaboram com a base bibliográfica, que defende que se deve propiciar ao longo da vida e, principalmente durante a senilidade, atividades cognitivamente e fisicamente 
estimulantes, de modo que se evite o retrocesso da cognição basal e das capacidades físicas como a locomoção (DUGAN et al., 2018; CUl et al., 2018; NELSON et al., 2007).

Entretanto, existe o "grupo", como denominam na clínica, que participa dos eventos da geriatria. Em geral os residentes mais ativos fisicamente e cognitivamente saudáveis participam (aproximadamente 20), fazem bolos na cozinha, aulas coletivas e participam de qualquer outro evento que surgir no local. Algo inusitado que se pode observar é que, como eles se cansam muito rápido e por vezes até dormem, a troca de participantes dentro do grupo durante uma atividade acontece seguidamente.

Quanto ao serviço prestado pelo profissional de Educação Física em si, pude observar as intervenções que ele executa no residencial, contabilizando cerca de 8 a 10 atendimentos por turno, ou 6 se for em residentes da UCE. As intervenções que os idosos eram submetidos que observei no período que permaneci lá em sua maioria eram:

a) Passeios nas redondezas da clínica (a maioria dos idosos que passeavam estavam na cadeira de rodas e poucas vezes levantavam-se para andar, somente me momentos estratégicos escolhidos pelo profissional): $O$ profissional sai com algum residente, geralmente na cadeira de rodas (pois a maioria não caminha mais do 20 a 30 passos), e executa um breve passeio na rua, que dura entre 10 a 15 minutos, a depender do morador e do clima;

b) Exercícios de mobilidade: os idosos mais debilitados ele os leva para a área de lazer/sala de ginástica que fica no térreo (ou faz intervenção no andar do paciente se este for muito debilitado) e os auxilia a caminhar, além de exercícios básicos de mobilidade articular com materiais como bolas de pilates ou até mesmo sem material.

O relato do profissional sobre essas intervenções é bem incisivo, ele diz que o principal objetivo da atuação dele junto aos moradores da clínica é evitar ao máximo que estes percam mobilidade, e fiquem ainda mais debilitados, pois sabe que uma melhora considerável nas morbidades (principalmente de cunho físico) dos mesmos é algo bem difícil, e o melhor a se fazer é evitar ou retardar essa degeneração articular, muscular e até mesmo cognitiva. 
Segundo o profissional esta é uma área de atuação muito pouco explorada pelos profissionais de Educação Física e que está em grande expansão, uma vez que a população pertencente à faixa etária da terceira idade está cada vez mais aumentando. Cabe ressaltar que o profissional atua em duas clínicas geriátricas, no turno da manhã (onde o observei) e outra no período da tarde.

Percebe-se então que o profissional de Educação Física é muito importante no contexto interdisciplinar da clínica, tanto para compor o grupo de intervenção em saúde do local, como no auxílio das atividades de mobilidade física com os residentes, pois muito além do exercício físico, o ato de mobilizar os indivíduos a realizarem atividades fora de suas rotinas, que em grande parte é insólita (deitar e sentar), é algo necessário trabalhando assim corpo e mente como indissociáveis.

Estudos elaborados por Baumgart et al. (2015) e a Alzheirmer's Association (2020), acreditam que há indícios fortemente estabelecidos de que fatores como a prática com certa regularidade de atividade física, a manutenção de fatores cardiovasculares, assim como a uma dieta saudável e um treinamento cognitivo de aprendizado escolar e não escolar, podem colaborar para um decréscimo do risco de declínio cognitivo.

Hewitt et al. (2018) ainda ressalva que a atividade física em indivíduos moradores de ILPIs é algo de grande importância, principalmente tendo em vista que com um aumento/manutenção da capacidade física neste período senil se pode evitar um enorme número de agravantes em decorrência da idade biológica, como as quedas constantes, os riscos de fraturas, a osteoporose e a sarcopenia.

De acordo com o The Lancet Comissions (2017) existem nove fatores modificáveis de risco para a demência e degeneração, principalmente na terceira idade (mais de 65 anos), os principais fatores que se deve evitar são Tabagismo, depressão tardia, diabetes, isolamento social e inatividade física (LIVINGSTON et al., 2017). Contribuir de alguma forma para modificar ou até mesmo evitar a progressão dessas doenças através da prevenção por meio da AF pode beneficiar no processo de envelhecimento com uma melhor autonomia e qualidade de vida.

A Segundo a Organização Mundial da Saúde (OMS) (2019), em seu relatório de recomendações, também esclarece quanto a esses fatores mutáveis de risco, sugerindo que para que se evitem as patologias que causam demências, se instaurem hábitos como: a prática regular de atividade física, não fumar (tabagismo), bem como o controle da hipertensão e do diabetes. 
É perceptível então, nos mais diversos estudos e relatórios internacionais, a repetição de alguns fatores em comuns, principalmente de aspectos educacionais (cognitivos), atividade física regular e aspectos cardíacos (como o controle da pressão arterial em pacientes hipertensos). Fatores esses que se cuidados podem contribuir em retardar o surgir da demência (principalmente a Doença de Alzheimer) ou até mesmo evitá-la (LOURENCO et al., 2019). Esses são alguns dos fatores principais para que se crie uma espécie de "reserva" de cognição, a reserva cognitiva, um constructo hipotético sobre o envelhecimento cognitivo que com base em uma inteligência inata do indivíduo e/ou experiências da vida pode-se minimizar o aparecimento das demências (SOBRAL; PESTANA; PAÚL, 2015).

\section{CONSIDERAÇÕES FINAIS}

O estágio realizado na clínica foi uma vivência ímpar, sem demagogia alguma, de verdade, nunca tivera presenciado tal realidade. Uma das coisas que mais me chama à atenção neste relato é o fato de que todos que ali residiam eram pessoas de "renome" na sociedade capitalista, e mesmo assim em contrapartida hoje muitos deles estão abandonados pelos familiares, tornando-se apenas mais um "morador de asilo", o que entristece muito, me fazendo refletir sobre a vida, pois hoje somos saudáveis, amanhã poderemos não ter o envelhecimento bem-sucedido (caso não nos cuidarmos ao longo da vida), ficando, como muitos observados, em situação de desleixo familiar, situação esta que não desejo para ninguém.

Em suma, através da experiência no Residencial Com vivência a multidisciplinaridade é importante para a formação de um profissional da saúde não somente da área de Enfermagem, Fisioterapia ou Educação Física, mas para todos da área, pois é uma experiência enriquecedora como ser humano, além de abrir os horizontes da atuação profissional posteriormente. A importância deste relato encontra-se além da apresentação desta realidade de abandono familiar, mas também serve para os profissionais da saúde, em especial os pertencentes a área da Educação Física, alertando para a existência deste campo de atuação pouco explorado até o momento. Finalizo enfatizando que é de suma importância prestarmos atenção nas oportunidades não somente durante a formação, mas ao longo da vida profissional, e percebo que, para quem se interessar, essa é uma área de especialização profissional com grande potencial no futuro próximo. 


\section{REFERÊNCIAS}

ALZHEIMER'S ASSOCIATION. 2020 Alzheimer's Disease Facts and Figures. Alzheimers Dement, v. 16, p. $391-470,2020$.

ANDREASSEN, S. N.; BEN EZRA, M.; SCHEIBYE-KNUDSEN, M. A defined human aging phenome. Aging (Albany NY), v. 11, n. 15, p. 5786 - 5806, 2019.

BAUMGART, M.; SNYDER, H. M.; CARRILLO, MC et al. Summary of the evidence on modifiable risk factors for cognitive decline and dementia: A populationbased perspective. Alzheimers Dement, v. 11, n. 6, p. 718-726, 2015.

BRASIL. Secretaria de Vigilância em Saúde. Secretaria de Atenção à Saúde. Política Nacional de Promoção da Saúde. Brasília: Ministério da Saúde, 2006a.

CUI, M. Y.; LIN, Y.; SHENG, J. Y. et al. Exercise Intervention Associated with Cognitive Improvement in Alzheimer's Disease. Neural Plasticity, v. 1, p. 01-10, 2018.

DI LIEGRO, C. M.; SCHIERA, G.; PROIA, P.; DI LIEGRO, I. Physical Activity and Brain Health. Genes (Basel), v. 10, n. 9, p. 720-760, 2019.

DUGAN, S. A.; GABRIEL, K. P.; LANGE-MAIA, B. S. et al. Physical Activity and Physical Function: Moving and Aging. Obstetrics and Gynecology Clinics of North America, v. 45, n. 4, p. 723-736, 2018.

DZIECHCIAŻ, M.; FILIP, R. Biological psychological and social determinants of old age: bio-psycho-social aspects of human aging. Annals of Agricultural and Environmental Medicine, v. 21, n. 4, p. 835-838, 2014.

GUTHOLD, R.; STEVENS, G. A.; RILEY, L. M.; BULL, F. C. Worldwide trends in insufficient physical activity from 2001 to 2016: a pooled analysis of 358 population-based surveys with 1. 9 million participants. Lancet Global Health, v. 6, n. 10, p. 1077-1086, 2018.

HEWITT, J.; GOODALL, S.; CLEMSON, L.; HENWOOD, T.; REFSHAUGE, K. Progressive Resistance and Balance Training for Falls Prevention in LongTerm Residential Aged Care: A Cluster Randomized Trial of the Sunbeam Program. Journal of the American Medical Directors Association, v. 19, n. 4, p. 361369, 2018.

INSTITUTO BRASILEIRO DE GEOGRAFIA E ESTATÍSTICA (IBGE). Pesquisa Nacional de Saúde (PNS) 2020. Disponível em: <https://www.ibge.gov.br/>. Acesso em: 28 mai. 2020.

INSTITUTO BRASILEIRO DE GEOGRAFIA E ESTATÍSTICA (IBGE). Pesquisa Nacional por Amostra de Domicílios Contínua (PNAD) 2020. Disponível em: <https://www.ibge.gov.br/>. Acesso em: 28 mai. 2020. 
LIVINGSTON, G. et al. The Lancet Comissions Dementia prevention, intervention, and care. The Lancet, v. 390, p. 2673-2734, 2017.

LOURENÇO, M. V.; FROZZA, R. L.; DE FREITAS, G. B. et al. Exercise-linked FNDC5/irisin rescues synaptic plasticity and memory defects in Alzheimer's models. Nature Medicine, v. 25, p. 165-175, jan., 2019.

NELSON, M. E.; REJESKI, W. J.; BLAIR, S.N. et al. Physical activity and public health in older adults: recommendation from the American College of Sports Medicine and the American Heart Association. Circulation, v. 166, n. 9, p. 10941105, 2007.

ORGANIZAÇÃO MUNDIAL DA SAÚDE (OMS). Relatório Mundial de Envelhecimento e Saúde. Genebra, 2015. Disponível em: $<$ https://www.who.int/ageing/events/world-report-2015-launch/en/>. Acesso em: 28 mai. 2020.

ORGANIZAÇÃO MUNDIAL DA SAÚDE (OMS). Redução do risco de declínio cognitivo e demência. Genebra, 2019.

OKUMA, S. S. O significado da atividade física para o idoso: um estudo fenomenológico. Tese (Doutorado) - Instituto de Psicologia, Universidade de São Paulo, 1997.

SOBRAL, Margarida; PESTANA, Maria Helena; PAUL, Constança. Cognitive reserve and the severity of Alzheimer's disease. Arquivos de Neuro-Psiquiatria, São Paulo, v. 73, n. 6, p. 480-486, Junho 2015.

WOLFF, Suzana Hubner (org). Vivendo e envelhecendo: Recortes de práticas nos núcleos de vida saudável. São Leopoldo: Editora Unisinos, 2009. 